\title{
Host age influence on the parasitism of the species Cotesia vestalis (Haliday) (Hymenoptera: Braconidae)
}

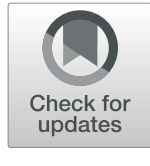

\author{
A. Saini, P. L. Sharma* and R. S. Chandel
}

\begin{abstract}
The parasitoid species Cotesia vestalis (Haliday) (Hymenoptera: Braconidae) is an important parasitoid, mass multiplied and used against the diamondback moth Plutella xylostella (Linneaus) (Lepidoptera: Plutellidae) worldwide. The present study was carried out to investigate the effect of host larval instars on parasitism and fitness to determine the most suitable host age for mass multiplication and augmentative releases of the parasitoid. Host age had a significant impact on the fitness of the parasitoid. Although C. vestalis was able to parasitize all stages of P. xylostella, it preferred the 2nd instar larvae. The biological and population growth parameters such as survival, fecundity, intrinsic rate of increase, finite rate of increase, mean generation length and weekly multiplication rate were higher when parasitism was on the 2nd instar larvae than the others. Therefore, the 2nd instar larvae are recommended for the mass production of highquality individuals of the parasitoid.
\end{abstract}

Keywords: Cotesia vestalis, Plutella xylostella, Life table, Biology

\section{Background}

The diamondback moth, Plutella xylostella (Linneaus) (Lepidoptera: Plutellidae), is one of the most important cosmopolitan insect pests of cruciferous crops throughout the world (Cobblah et al. 2012). Cabbage, cauliflower, broccoli, Brussel sprout, Chinese cabbage, mustard, radish, etc. are the economically important crops attacked by this pest. P. xylostella causes 50 to $90 \%$ 'avoidable' yield losses depending upon the crop and the infestation level (Oke, 2008). The pest is particularly notorious for its resistance to insecticides including Bacillus thuringiensis Berliner (Sarfraz and Keddie, 2005, Ishadi and Mazlan, 2015). Under such circumstances, the use of bio-control agents, especially the parasitoids, is an eco-friendly alternative control method. Among the parasitoids, Diadegma semiclausum (Hellen) (Hymenoptera: Ichneumonidae), D. rapi (Cameron) (Hymenoptera: Ichneumonidae), D. mollipla (Holmgren) (Hymenoptera: Ichneumonidae), Cotesia plutellae (Kurdjumov) (= Cotesia vestalis (Haliday) (Hymenoptera: Braconidae), Diadromus collaris (Grav.) (Hymenoptera: Ichneumonidae) and Oomyzus sokolowskii (Kurdjumov)

\footnotetext{
* Correspondence: Sharma.pl@rediffmail.com

Department of Entomology, YSP University of Horticulture and Forestry, Nauni, Solan, Himachal Pradesh 173 230, India
}

(Hymenoptera: Eulophidae) have been reported (Sadia et al., 2015).

C. vestalis, a synovigenic arrhenotokous braconid, is one of the dominant larval parasitoids, causing 16 to $70 \%$ larval parasitism of $P$. xylostella in different parts of the world (Seenivasagan et al., 2010). The parasitoid is native to the warmer areas of the Palearctic but has been introduced to other parts of the world for the control of this pest (Furlong et al., 2013). The parasitoid is able to parasitize all the larval instars of $P$. xylostella (Kawaguchi and Tanaka, 1999).

The success of a parasitoid as an efficient bio-control agent depends on its fitness (survival, fecundity, development duration, sex ratio, etc.) over generations. The intrinsic rate of natural increase is a measure of the biotic potential of the species and the advantage of using this measure is that it integrates the effect of the fertility factors into a single value.

Very little is known about the host age that parasitoid prefers for oviposition and the impact of the host developmental stage on the fitness parameters of the parasitoid. Knowledge of the most suitable/preferred host age by the parasitoid is also crucial for augmentative releases of the parasitoid ( $\mathrm{Li}$ et al., 2006). Use of most suitable 
host age for mass rearing of the parasitoid will certainly improve the fitness parameters of the parasitoid in terms of survival, fecundity and development time. The present study was carried out to evaluate the impact of developmental larval ages of $P$. xylostella on the biological parameters of $C$. vestalis to optimize the parasitoid's mass rearing process.

\section{Materials and methods}

The present study was carried out in the Bio-control Laboratory of the Department of Entomology, University of Horticulture and Forestry, Nauni, Solan, Himachal Pradesh, India. Experiments were conducted at $25 \pm 0.5^{\circ} \mathrm{C}, \quad 70 \pm 5 \%$ relative humidity and $12 \mathrm{~L}: 12 \mathrm{D}$ photoperiod.

\section{Insect cultures}

\section{Plutella xylostella}

A stock culture of $P$. xylostella was established at $25 \pm 0.5^{\circ} \mathrm{C}, 70 \pm 5 \% \mathrm{RH}$ and $12 \mathrm{~L}: 12 \mathrm{D}$ photoperiod in the laboratory from field collected larvae. The larvae were fed on cauliflower (Brassica oleracea var botrytis L.) leaves or mustard (Brassica campestris L.) seedlings in insect rearing cages $(45 \times 45 \times 45 \mathrm{~cm})$ fitted with glass on 3 sides and a nylon net on the front side. Larval food was changed daily or on alternate days (depending upon the requirement) until all the larvae pupated. Pupae were collected and placed in jars for adult emergence. Newly emerged adults were shifted to the insect rearing cages for mating and provided with $30 \%$ honey solution (in cotton swabs) as food and fresh leaves of cauliflower with their petioles inserted in a glass/plastic tube containing water as substrate for oviposition. After $24 \mathrm{~h}$, the leaves with eggs were shifted to another cage for hatching. Neonate larvae were transferred carefully to the fresh cauliflower leaves for further rearing under laboratory conditions. P. xylostella was reared and multiplied for 2 generations before experiments were carried out.

\section{Cotesia vestalis}

A culture of $C$. vestalis (national accession number: NBAII-GN-BRA-04) was maintained from cocoons, obtained from the National Bureau of Agricultural Insect Resources, Bengaluru, Karnataka, India. The emerged adults were transferred to rearing cages $(45 \times 45 \times 45 \mathrm{~cm}$, fitted with glass on 3 sides and a nylon net on the front side) and provided with $30 \%$ honey solution (in cotton swabs) for feeding and the 2 nd $/ 3$ rd instar larvae of $P$. xylostella (on cauliflower leaves) for oviposition. After $24 \mathrm{~h}$, the larvae were removed and a new batch of larvae was provided to the parasitoids. The process was repeated till all the parasitoids died. Parasitized larvae of P. xylostella were reared at $25 \pm 0.5^{\circ} \mathrm{C}, 70 \pm 5 \% \mathrm{RH}$ and
12L:12D photoperiod. The food of the adult parasitoid was renewed daily. C. vestalis was reared for 2 generations to obtain a sufficient number of parasitoids for the experiments.

\section{Preference of $C$. vestalis for different larval stages of $P$. xylostella}

To study the relative preference of C. vestalis for different larval ages of $P$. xylostella, parasitoid adults were provided by all the larval instars of $P$. xylostella simultaneously. Newly emerged parasitoid adults were sexed and each pair was confined in a glass tube $(15 \times 2.5 \mathrm{~cm})$ containing honey streak on the side wall for $24 \mathrm{~h}$ to ensure mating. After $24 \mathrm{~h}$, in a choice experiment, a pair of $C$. vestalis was provided by 20 larvae (replicated 5 times), 5 each of the 1st, 2nd, 3rd and 4th instars of the host on a cauliflower leaf simultaneously in a rearing cage. Larval instars of P. xylostella were differentiated on the bases of their head capsule width, body colour and size (Alizadeh et al., 2011). The parasitized host larvae of different ages were reared separately as described earlier until pupation and then adult emergence. The data on the number of parasitized larvae for each instar were recorded.

\section{Developmental biology of C. vestalis parasitizing different host instars}

Developmental biology of $C$. vestalis was studied by offering different larval instars of $P$. xylostella for parasitization. For this purpose, newly emerged parasitoid adults were sexed and each pair was offered by 10 larvae of the 2 nd, 3rd or 4th instar larvae of the host insect separately in a no-choice experiment. After $24 \mathrm{~h}$, the parasitized larvae were replaced with a new batch of ten larvae. The parasitized larvae were reared as per the procedure described above for the emergence of the parasitoids. Because the egg and larval stages of $C$. vestalis developed inside the body of the larva of $P$. xylostella, egg and larval instars were combined as egg-larval stage. Data on the duration of egg + larva (stinging to cocoon formation), prepupa + pupa (cocoon stage), adult longevity, sex ratio and fecundity (in terms of progeny developed) were recorded.

\section{Population growth parameters of $C$. vestalis parasitizing different host instars}

Population growth parameters of $C$. vestalis on $P$. xylostella were measured, using fertility tables. Fifty larvae of each instar parasitized by $C$. vestalis on a single day were separated and reared as described earlier. To obtain parasitized larvae, individual larva of $P$. xylostella was exposed to a 24-h-old mated female parasitoid in a glass tube and replaced with 
another one after being stung once by the parasitoid. In this way, nearly 20 parasitized larvae per hour were obtained. The female of $C$. vestalis was replaced after stinging 5 larvae. Host larva stung by the parasitoid was presumed to be parasitized. Adults thus obtained were sexed and each couple of parasitoid adults was offered by 20 host larvae of the desired age. After $24 \mathrm{~h}$, the old batch of larvae was replaced by a new batch and the process was continued until all the parasitoids died. Daily survival and fecundity data were used to construct fertility-based life tables of the parasitoid on each host instar separately to calculate the population growth parameters (Birch 1948 and Carey 2001) as follows:

Net reproductive rate $\left(R_{o}\right)=\Sigma l x m x$.

Intrinsic rate of increase $\left(r_{m}\right)$ was calculated by using the expression $\Sigma\left(e^{-r m x} l x m x\right)=1$.

Mean generation time $(T)=\log _{\mathrm{e}} R_{o} / r_{m}$.

Finite rate of natural increase $(\lambda)=$ Antilog $_{\mathrm{e}} r_{m}$.

Weekly multiplication rate $(\mathrm{WM})=e^{7 r m}$

Doubling time (DT) $=\log _{\mathrm{e}} 2 / r_{m}$.

Where $x=$ age of the individuals in days (pivotal age), $l x=$ the proportion of females still alive at age $x$ (survival rate) and $m x=$ the number of female eggs (based on sex ratio) per female at the age $x$ (fecundity rate).

The jack-knife method was used to generate pseudoreplication for obtaining standard errors of parameters with 5 replications in each case (Maia et al. 2000, Meyer et al. 1986).

\section{Statistical analysis}

Data on different parameters were subjected to oneway analysis of variance (ANOVA), using OP-STAT available at http://www.hu.ernet.in., and the significantly different means were separated by least significant difference (LSD).

\section{Results and discussion}

\section{Host age preference}

There were significant differences in $C$. vestalis parasitization rates among different larval instars of $P$. xylostella, when they were offered simultaneously to the parasitoid $(p<0.01)$. Although the parasitoid could parasitize the entire host instars, it preferred the 2nd instar (84\%), followed by the 3rd instar $(52 \%)$. The 1 st and 4 th instar larvae were less preferred by the parasitoid with 8 and 20\% parasitism, respectively (Table 1 ). Second instar larvae were preferred more consistently (coefficient of variation $(\mathrm{CV})=10.6)$ than other ages $(\mathrm{CV}=21.1,70.7$ and 136.9 for III, IV and I instars, respectively).
Table 1 Preference of $C$. vestalis for different larval stages of $P$. xylostella

\begin{tabular}{llll}
\hline Host instar & $\begin{array}{l}\text { Mean ( } \pm \text { SE) number of larvae } \\
\text { parasitized out of five larvae } \\
\text { of each instar }\end{array}$ & CV (\%) & Parasitization (\%) \\
\hline I & $0.4 \pm 0.2^{c}$ & 136.9 & 8 \\
II & $4.2 \pm 0.2^{\mathrm{a}}$ & 10.6 & 84 \\
III & $2.6 \pm 0.2^{\mathrm{b}}$ & 21.1 & 52 \\
IV & $1.0 \pm 0.3^{\mathrm{c}}$ & 70.7 & 20 \\
$\mathrm{CD}(p=0.05)$ & 0.7 & &
\end{tabular}

Values superscripted by the same letter in the column do not differ significantly at $p \leq 0.05$

\section{Developmental biology of $C$. vestalis parasitizing different} host ages

For parasitization, larval instar significantly affected the survival to adult emergence and fecundity (survival to adult emergence: $p<0.001$; fecundity: $p<0.001$ ) (Figs. 1 and 2). The duration of the entire immature stages (stinging of the host to adult emergence) from the host parasitized in the 2nd, 3rd and 4th instars was not statistically different from each other $(p=0.08)$ (Table 2). The particular host age that was parasitized had insignificant effect on the longevity of the parasitoid (male longevity: $p=0.30$; female longevity: $p=0.09$ ) (Table 2 ). Daily survival and fecundity was highest for the parasitoid starting parasitism in the 2nd instar larvae of the host, followed by 3rd and 4th instars (Table 3 and Figs. 1 and 2). Sex ratio was male biased in all the cases and varied from $45.5 \%$ females and $54.5 \%$ males when host was parasitized in the 4th instar to $40 \%$ females and $60 \%$ males when parasitism occurred in the 3rd instar of the host larvae.

\section{Population growth parameters of $C$. vestalis parasitizing different host ages}

Highest values $r_{m}$ (0.207), $R_{o}$ (48.3) and $\lambda$ (1.23) were achieved when parasitism begun in the 2nd instar of the host as compared to the 3rd or 4th instar (Table 3). With these parameters, the parasitoid could double its population in 3.4, 4.5 and 7.2 days with a weekly multiplication rate of 4.25, 2.97 and 1.95 when parasitism took place in the 2nd, 3rd and 4th instar larvae, respectively. The mean generation length of the parasitoid varied from 18 to 18.9 days and was similar irrespective of which host age was parasitized.

Selection of a high-quality bio-control agent is crucial for any successful bio-control programme. Earlier studies indicated that the fitness of the parasitoid was influenced by the age of the host insect and, hence, can be improved by selecting the most suitable age for mass rearing of the parasitoid ( $\mathrm{Li}$ et al., 2006). 


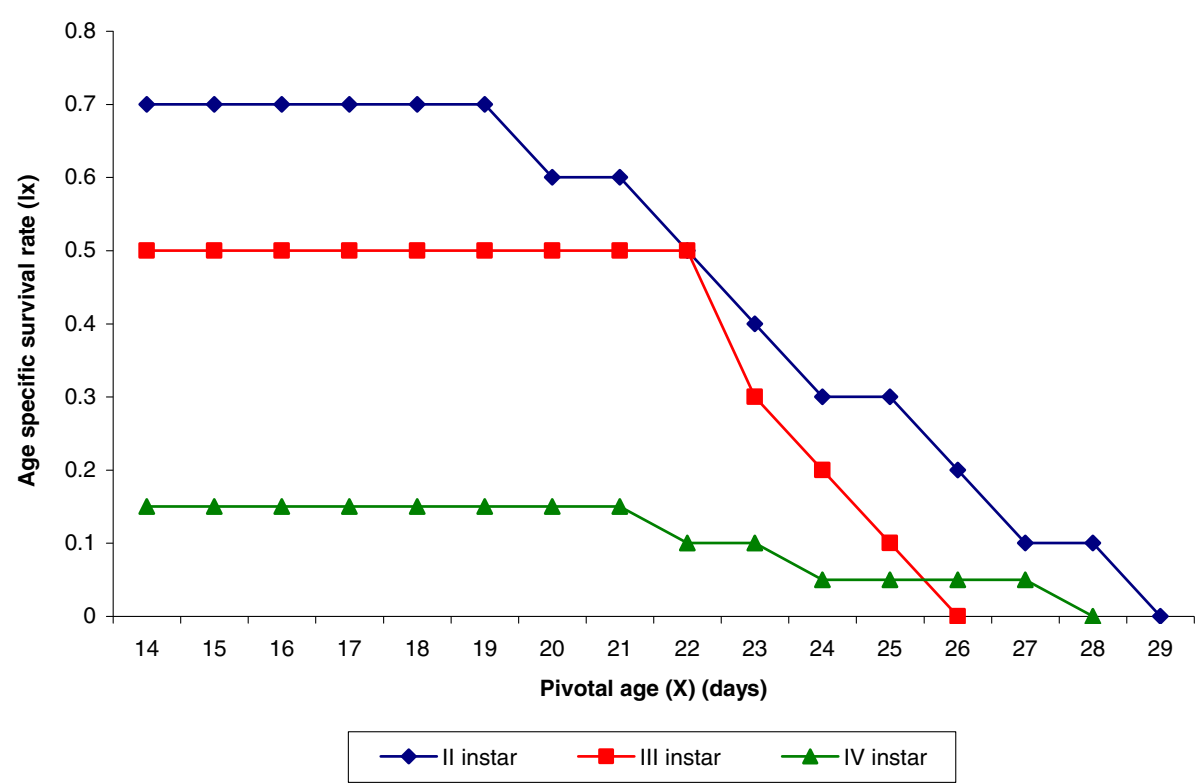

Fig. 1 Age-specific survival of Cotesia vestalis on different host instars

Most parasitoids can distinguish between a suitable and unsuitable host and, therefore, can make a selection of the appropriate host age for oviposition ( $\mathrm{Li}$ et al., 2006 and Murillo et al., 2013). Obtained results showed that $C$. vestalis preferred to parasitize the 2 nd instar larvae of $P$. xylostella (Alizadeh et al., 2011). However, Shi et al. (2002) reported equal preference of the parasitoid for the 3rd and 2nd instar host larvae. The results agree with a number of previous studies (Shi et al., 2002, Alizadeh et al., 2011). Based on these studies, the preference of C. vestalis to host (except the 1st instar) decreased with the host age.

The development of immature stages obtained in the present study is similar to that reported by $\mathrm{Yu}$ et al. (2008). Although the developmental period of immature stage of $C$. vestalis parasitizing the 2 nd instar larvae of $P$. xylostella was slightly longer than that on other instars, the 2nd instar larvae were the most

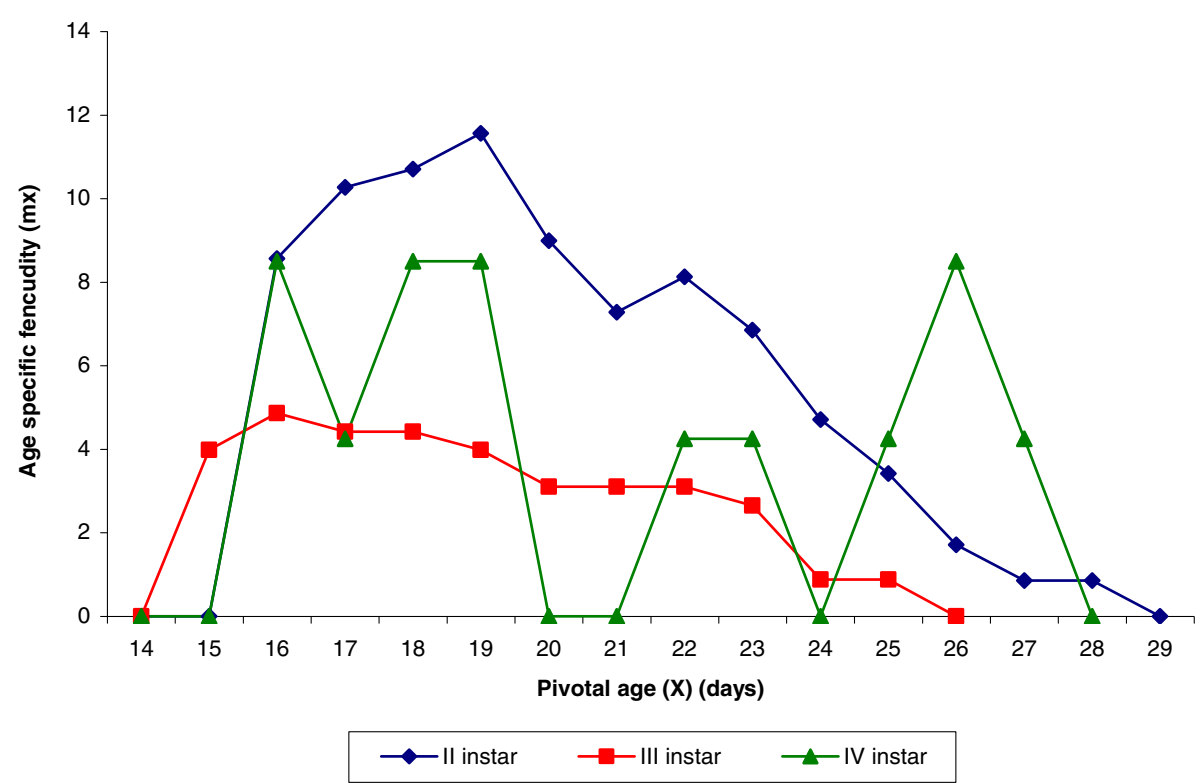

Fig. 2 Age-specific fecundity $(m x)$ of Cotesia vestalis on different host instars 
Table 2 Developmental period of C. vestalis starting parasitism in the II, III and IV instars of P. xylostella

\begin{tabular}{|c|c|c|c|c|c|}
\hline \multirow{3}{*}{$\begin{array}{l}\text { Host } \\
\text { instar }\end{array}$} & \multicolumn{5}{|c|}{ Mean development time (days \pm SE) } \\
\hline & \multirow{2}{*}{$\begin{array}{l}\text { Stinging to } \\
\text { cocoon } \\
\text { formation }\end{array}$} & \multirow{2}{*}{$\begin{array}{l}\text { Cocoon } \\
\text { stage }\end{array}$} & \multirow{2}{*}{$\begin{array}{l}\text { Stinging to } \\
\text { adult emergence }\end{array}$} & \multicolumn{2}{|c|}{ Adult longevity } \\
\hline & & & & Male & Female \\
\hline & $8.2 \pm 0.4^{a}$ & $6.8 \pm 0.2^{a}$ & $15.0 \pm 0.5^{a}$ & $9.8 \pm 0.4^{a}$ & $11.8 \pm 0.5^{\mathrm{a}}$ \\
\hline 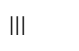 & $7.4 \pm 0.2^{\mathrm{a}}$ & $6.1 \pm 0.3^{\mathrm{a}}$ & $13.4 \pm 0.2^{\mathrm{a}}$ & $10.0 \pm 0.3^{\mathrm{a}}$ & $11.8 \pm 0.4^{\mathrm{a}}$ \\
\hline & $8.0 \pm 0.3^{a}$ & $6.2 \pm 0.3^{\mathrm{a}}$ & $14.0 \pm 0.5^{\mathrm{a}}$ & $9.8 \pm 0.4^{a}$ & $11.3 \pm 0.6^{a}$ \\
\hline
\end{tabular}

Values superscripted by the same letter in the column do not differ significantly at $p \leq 0.05$

suitable stage for the parasitoid fitness (survival and fecundity). Logically, a bigger host is expected to provide better nutrition to the developing parasitoid larva (Li et al., 2006), and there are reports that parasitoids such as Microplitis demolitor Wilkinson (Hymenoptera: Braconidae), Pseudaphycus maculipennis Mercet (Hymenoptera: Encyrtidae) and Diaeretiella rapae (M'Intosh) (Hymenoptera: Draconidae) prefer bigger hosts over the smaller ones (Harvey et al., 2004). Contrary to this, the preference of $C$. vestalis to select a host for oviposition decreased with the increase in the host size. In fact, host size was not the only criteria for selection by the parasitoid; other factors like nutritional quality and quantity, host's defence and endocrine changes may influence the host selection by the parasitoid (Li et al., 2006, Murillo et al., 2013). Solitary endoparasitoids choose slowly growing larvae for oviposition and regulate or slow down the host development in their own favour (Khafagi and Hegazi, 2004 and Chu et al. 2014). This ability of the parasitoid could probably enable it to complete successfully the development in nearly the same time even when it attacks the last larval instar as has also been observed in the present study. Sex ratio is another criterion for the parasitoid fitness and it was male biased in all cases, which confirm the findings of Alizadeh et al. (2011). The adult longevity of the parasitoid recorded in the present study was higher than that reported by Nofemela (2004) and lower than reported by Alizadeh et al. (2011). The differences in the longevity of $C$. vestalis recorded in these studies could be due to the differences in the geographical populations of $C$. vestalis and/or $P$. xylostella used. The parasitoid females ovipositing in the 2nd instar host larvae were more fecund than those ovipositing in the 3rd and 4th host instars. These results differ slightly from those of $\mathrm{Yu}$ et al. (2008), where the females starting parasitism in the 3rd instar larvae of $P$. xylostella had higher fecundity than other instars.

Fertility tables summarized the information on biological performance of the species taking into consideration, the age-specific survival and fecundity of the species to determine the net reproductive rate $\left(R_{o}\right)$ and intrinsic rate of natural increase $\left(r_{m}\right)$. Population growth parameters such as intrinsic rate of increase, net reproductive rate and finite rate of increase are influenced by nature and quality of the host (Farhadi et al., 2011). In the present study, the values of intrinsic rate of increase, net reproductive rate and finite rate of increase of $C$. vestalis were highest when it parasitized the 2nd instar and lowest when it parasitized the 4th instar. In addition, the parasitoid initiating parasitism in the 2nd instar larvae of the host would require less time to double its population than that initiating parasitism in the 3rd or 4th instar larvae of the host. Therefore, it can be inferred that the 2nd instar larvae of $P$. xylostella was the most suitable age for oviposition to produce the fittest progeny of the parasitoid.

\section{Conclusion}

C. vestalis could parasitize all the larval stages of $P$. xylostella with preference for the 2nd instar. Biological and population growth parameters of the parasitoid were better when parasitism took place in the 2nd instar host larvae than other stages. Selection of a suitable host age is crucial for mass production of a parasitoid and its utilization in augmentative biological control.

Table 3 Population growth parameters of $C$. vestalis parasitizing different larval instars of $P$. xylostella

\begin{tabular}{|c|c|c|c|}
\hline \multirow[t]{2}{*}{ Parameter } & \multicolumn{3}{|l|}{ Larval instar } \\
\hline & $\|$ & III & IV \\
\hline Net reproductive rate $\left(\underline{R_{0}}\right)$ (female progeny/female) & $48.3 \pm 3.8^{\mathrm{a}}$ & $16.5 \pm 1.6^{b}$ & $6.2 \pm 1.9^{c}$ \\
\hline True intrinsic rate of increase $\left(r_{m}\right)$ (females/female/day) & $0.207 \pm 0.008^{\mathrm{a}}$ & $0.156 \pm 0.004^{b}$ & $0.096 \pm 0.01^{c}$ \\
\hline True generation time $(T)$ (days) & $18.8 \pm 1.4^{\mathrm{a}}$ & $18.0 \pm 1.1^{\mathrm{a}}$ & $18.9 \pm 1.9^{\mathrm{a}}$ \\
\hline Finite rate of natural increase $(\lambda)$ (females/day) & $1.230 \pm 0.02^{\mathrm{a}}$ & $1.168 \pm 0.05^{b}$ & $1.101 \pm 0.08^{c}$ \\
\hline Doubling time (DT) (days) & $3.4 \pm 0.3^{\mathrm{a}}$ & $4.5 \pm 0.2^{b}$ & $7.2 \pm 0.5^{c}$ \\
\hline Weekly multiplication rate (WM) (folds) & $4.25 \pm 0.48^{\mathrm{a}}$ & $2.97 \pm 0.24^{b}$ & $1.95 \pm 0.26^{c}$ \\
\hline
\end{tabular}

Values superscripted by the same letter in the row do not differ significantly at $p \leq 0.05$ 


\section{Abbreviations}

ANOVA: Analysis of variance; CD: Critical difference; CV: Coefficient of variation; LSD: Least significant difference

\section{Acknowledgements}

The authors are thankful to the Indian Council of Agricultural Research, New Delhi, India, for providing funds through All India Coordinated Research Project on Biological Control of Crop Pests and Weeds.

\section{Authors' contributions}

SPL planned the research and designed the methodology. SA conducted the experiments and analysed the data, and CRS drafted the manuscript. All the authors have read and approved the final manuscript.

\section{Funding}

The study was funded by the Indian Council of Agricultural Research, New Delhi, India.

\section{Availability of data and materials}

Not applicable

\section{Ethics approval and consent to participate}

Not applicable

\section{Consent for publication}

Not applicable

\section{Competing interests}

The authors declare that they have no competing interests.

Received: 8 April 2019 Accepted: 22 July 2019

Published online: 02 August 2019

\section{References}

Alizadeh M, Rassoulian GR, Karimzadeh J, Naveh H, Farazmand H (2011) Biological study of Plutella xylostella (L.) (Lepidoptera: Plutellidae) and its solitary endoparasitoid, Cotesia vestalis (Haliday) (Hymenoptera: Braconidae) under laboratory conditions. Pakistan J Biol Sci 14:1090-1099

Birch LC (1948) The intrinsic rate of natural increase in an insect population. J An Ecol 17:15-26

Carey JR (2001) Insect biodemography. Ann Rev Entomol 46:79-110

Chu Y, Michaud JP, Zhang J, Li Z, Wang Y, Chen H, Li J, Lu Z, Zhang Q, Liu X (2014) Performance of Microplitis tuberculifer (Hymenoptera: Braconidae) parasitizing Mythimna separate (Lepidoptera: Noctuidae) in different larval instars. Biol Cont 69:18-23

Cobblah MA, Nuamah KA, Wilson D, Osae MY (2012) Parasitism of Plutella xylostella (L.) (Lepidoptera: Plutellidae) population on cabbage, Brassica oleracea var. capitata (L.) by Cotesia plutellae (Kurdjumov) (Hymenoptera: Braconidae) in Ghana. West African J Appl Ecol 20:37-45

Farhadi R, Allahyari H, Chi H (2011) Life table and predation capacity of Hippodamia variegata (Coleoptera: Coccinellidae) feeding on Aphis fabae (Hemiptera: Aphididae). Biol Cont 59:83-89

Furlong MJ, Wright DJ, Dosdall LM (2013) Diamondback moth ecology and management: problems progress and prospects. Ann Rev Entomol 58:517541

Harvey JA, Bezemer TM, Elzinga A, Strand MR (2004) Development of the solitary endoparasitoid Microplitis demolitor: Host quality does not increase with host age and size. Ecol Entomol 29:35-43

Ishadi A, Mazlan N (2015) Insecticide use impacts on pest resistance: an evidence from diamondback moth. Int J Sci: Basic and Appl Res 22:131-150

Kawaguchi M, Tanaka T (1999) Biological characteristics of larval endoparasitoid, Cotesia plutellae (Hymenoptera: Braconidae): host age preference, subsequent ratio of progeny and mate location of males. J Appl Entomol 34:213-221

Khafagi WE, Hegazi EM (2004) Is the host or the parasitoid in control? Effects of host age and temperature on pseudoparasitization by Microplitis rufiventris inSpodoptera litoralis. J Insect Physiol 50:577-584

Li JC, Coudron TA, Pan WL, Liu XX, Lu ZY, Zhang QW (2006) Host age preference of Microplitis mediator (Hymenoptera: Braconidae) and endoparasitoid of Mythimna separata (Lepidoptera: Noctuidae). Bioll Cont 39:257-261
Maia AHN, Luiz AJB, Campanhola C (2000) Statistical inference on associated fertility life table parameters using jackknife technique: computational aspects. J Econ Entomol 93:511-518

Meyer JS, Ingersoll CG, McDonald LL, Boyce MS (1986) Estimating uncertainty in population growth rates: jackknife vs. bootstrap techniques. Ecology 67: 1156-1166

Murillo H, Hunt DWA, VanLaerihoven SL (2013) Host suitability and fitness related parameters of Campoletis sonorensisi (Hymenoptera: Icneumonidae) as parasitoid of cabbage looper, Trichoplusia ni (Lepidoptera Noctuidae). Biol Cont 64:10-15

Nofemela S (2004) Studies on parasitoids of diamondback moth, Plutella xylostella (L.) (Lepidoptera: Plutellidae), in South Africa. M.Sc Thesis, Rhodes University, South Africa, p 36

Oke OA (2008) Evaluation of the effectiveness of three insecticides to control diamondback moth, Plutella xylostella (L.) in cabbage (Brassica Oleracea Var. (apitata). European J Sci Res 22:391-395

Sadia M, Lloyd M, John T (2015) Evolutionary ecology of diamondback moth, Plutella xylostella (L.) and Diadegma insulare (Cresson) in North America: A review. Ann Res Rev Biol 5:189-206

Sarfraz M, Keddie BA (2005) Conserving the efficacy of insecticides against Plutella xylostella (L.) (Lepidoptera: Plutellidae). J Appl Entomol 129:149-157

Seenivasagan T, Gandhi GR, Navarajan AV (2010) Differential parasitism by Cotesia plutellae (Kurdjumov) on Plutella xylostella (L.) in artificially infested host plants. J Biol Cont 24:22-27

Shi ZH, Liu SS, Li YX (2002) Cotesia plutellae parasitizing Plutella xylostella: Hostage dependent parasitism and its effect on host development and food consumption. J Biol Con 47:499-511

Yu RX, Shi M, Huang F (2008) Immature development of Cotesia vestalis (Hymenoptera: Braconidae), an endoparasitoid of Plutella xylostella (Lepidoptera: Plutellidae). Ann Entomol Soc America 101:189-196

\section{Publisher's Note}

Springer Nature remains neutral with regard to jurisdictional claims in published maps and institutional affiliations.

\section{Submit your manuscript to a SpringerOpen ${ }^{\circ}$ journal and benefit from:}

- Convenient online submission

- Rigorous peer review

- Open access: articles freely available online

High visibility within the field

- Retaining the copyright to your article

Submit your next manuscript at $>$ springeropen.com 